\title{
Single-fraction image-guided high-dose-rate brachytherapy for head and neck cancer: three cases of palliative brachytherapy
}

\author{
Yoshiaki Ota, MD, Koji Masui, MD, Gen Suzuki, MD, PhD, Hideya Yamazaki, MD, PhD, Kei Yamada, MD, PhD \\ Department of Radiology, Kyoto Prefectural University of Medicine, Kyoto, Japan
}

\begin{abstract}
Purpose: To present a small series of cases, in which single-fraction image-guided high-dose-rate (HDR) brachytherapy was provided as palliative treatment for inoperable head and neck cancer cases.

Material and methods: We used this technique to treat 3 patients with symptomatic head and neck cancer (tongue cancer, gingival metastasis of osteosarcoma, and external auditory canal cancer), who were not eligible for a definitive treatment. The purpose of palliative brachytherapy in all 3 patients was the relief of symptoms such as difficulty in eating, talking, and hearing. All patients were treated with computed tomography (CT) image-guided interstitial HDR brachytherapy under local anesthesia in an outpatient setting, with a single dose of $10 \mathrm{~Gy}$.

Results: Satisfactory palliative effects were achieved in all cases without serious side effects. The palliative effects were sustained in 2 patients until death, and 1 patient lived for 39 weeks before a relapse.

Conclusions: Our palliative treatment technique appears to be safe, effective, and less invasive, and could be a treatment option for symptomatic patients with head and neck cancer.

J Contemp Brachytherapy 2020; 12, 3: 273-278 DOI: https://doi.org/l0.5114/jcb.2020.96870
\end{abstract}

Key words: brachytherapy, palliative care, image-guided brachytherapy, head and neck neoplasms.

\section{Purpose}

Interstitial brachytherapy has historically been preferred for treatment of head and neck cancer [1,2]. Highdose-rate (HDR) brachytherapy is frequently performed as a part of treatment of these cancers $[3,4,5]$, but there have been few reports using HDR brachytherapy as a palliative treatment $[6,7,8]$. HDR brachytherapy has several advantages. For instance, the procedure allows administration of higher dose to the tumor in only few minutes, and the image-guided technique enables the delivery of higher dose to the tumor, but a lower dose to the surrounding tissue. Thus, image-guided HDR brachytherapy can be expected to be effective not only for radical therapy, but also for palliative treatment.

In this report, we describe 3 patients with head and neck cancer, who were not eligible for definitive treatment and whose palliative HDR brachytherapy was deemed effective. To our knowledge, no previous reports have documented the use of single-fraction image-guided HDR brachytherapy as a palliative treatment. We believe that it is valuable to share our experience in order to inform about the potential utility of HDR brachytherapy as palliative treatment.

\section{Patients}

In the 3 patients presented in this report, functional status was assessed using the Eastern Cooperative Oncology Group performance status (ECOG PS) scale. The scores were poor, suggesting poor prognosis. Patient 1 had systemic metastasis of esophageal cancer and tongue cancer, which caused difficulty in eating and talking. Patient 2 presented with systemic metastasis of osteosarcoma, with a right gingival metastasis causing difficulty in eating. Patient 3 had right hemiplegia due to previous cerebral infarction and had become completely deaf due to previous chronic otitis media in the left ear and external auditory canal cancer in the right ear (Table 1). None of the patients had any previous history of irradiation to the head and neck.

\section{Brachytherapy \\ Interstitial brachytherapy}

All patients were treated in an outpatient setting. Under local anesthesia, 1-8 applicators were placed with at least $1.5 \mathrm{~cm}$ space between them. Then, computed tomography $(\mathrm{CT})$ was performed to evaluate their locations and 
Table 1. Patients characteristics

\begin{tabular}{lccc} 
Parameter & Patient 1 & Patient 2 & Patient 3 \\
\hline Age (years) & 66 & 17 & 88 \\
\hline ECOG PS & 1 & 2 & 2 \\
\hline Co-morbidity & $\begin{array}{c}\text { Multiple metastasis of esophagus } \\
\text { cancer }\end{array}$ & $\begin{array}{c}\text { Multiple metastasis } \\
\text { of osteosarcoma }\end{array}$ & $\begin{array}{c}\text { Chronic otitis media of the right ear } \\
\text { with right sided-hemiplegia }\end{array}$ \\
\hline Pathology & SCC & Osteosarcoma & SCC \\
\hline Tumor location & Dorsum of tongue & Right gingiva behind the molar & Left auditory canal \\
\hline Symptoms & Dysphagia, dysarthria & Oral discomfort, & Deafness
\end{tabular}

ECOG PS - Eastern Cooperative Oncology Group performance status, SCC - squamous cell carcinoma

to complete treatment plans. CT images were obtained with 2.5-mm slice thickness.

\section{Planning}

In the treatment planning system (Oncentra ${ }^{\circledR}$ Brachy; Elekta Instrument AB, Stockholm, Sweden), we delineated target volume as gross tumor volume (GTV) and organs at risk (OARs), which were the mandible or inner
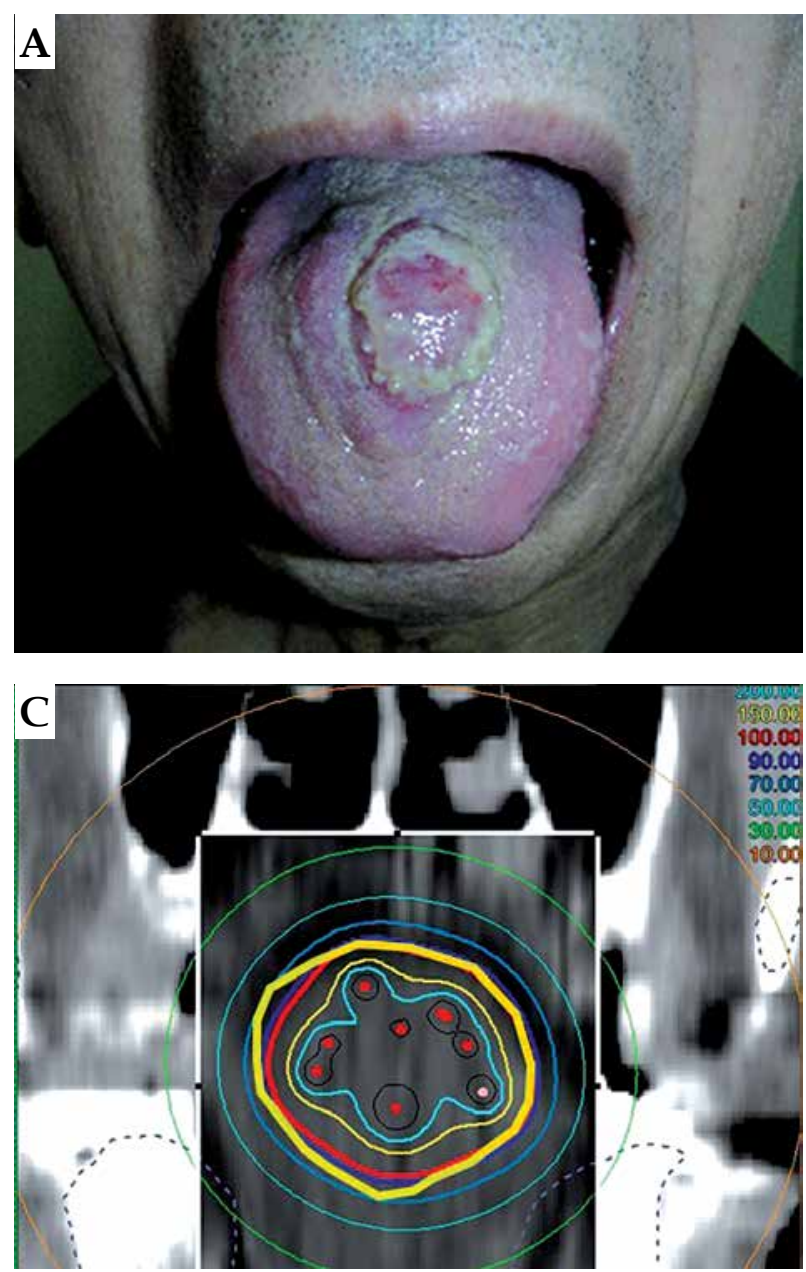

Fig. 1. Patient 1. A) Tongue cancer on the middle of dorsum linguae; B) Eight applicators placed inside the tumor with at least $1.5 \mathrm{~cm}$ space between them; C) Yellow circle indicates the contour of CTV; D) Tumor at 11 weeks after the treatment ear. We regarded the GTV as the clinical target volume (CTV). CT-based three-dimensional treatment planning was performed and evaluated the dose in relation to the CTV as well as the dose to OARs using dose-volume histograms. The prescribed dose was a single-fraction of $10 \mathrm{~Gy}$; we optimized the dose that would cover $90 \%$ of the CTV $\left[\mathrm{D}_{90}(\mathrm{CTV})\right]$ to $10 \mathrm{~Gy}$, and the minimum dose received by maximally irradiated $0.1 \mathrm{cc}$ volume of OARs $\left[D_{0.1 c c}(O A R s)\right]$ was less than $10 \mathrm{~Gy}$. We used MicroSelec-
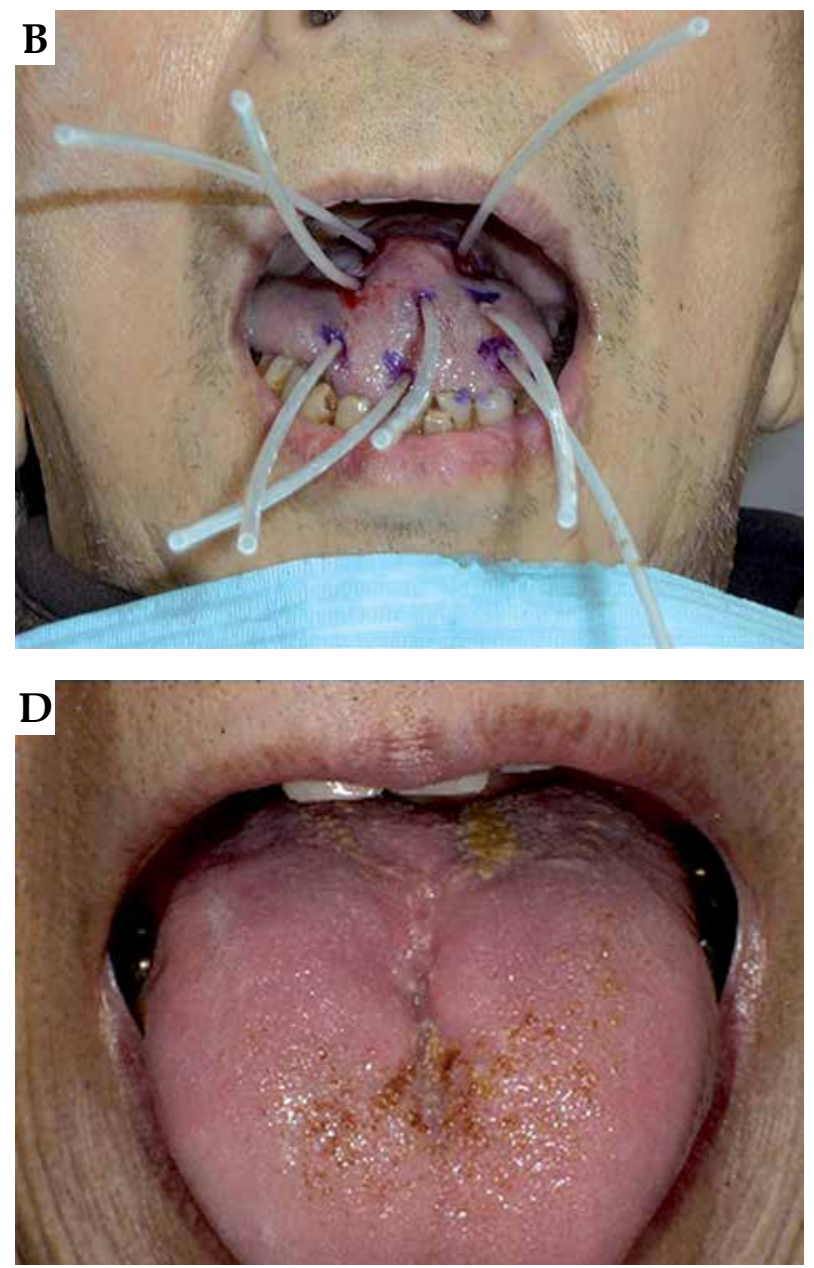
tron-HDR ${ }^{\circledR}$ Digital platform (Elekta AB, Stockholm, Sweden) for treatment and iridium-192 $\left({ }^{192} \mathrm{Ir}\right)$ as the treatment source.

\section{Case reports}

\section{Patient 1}

A 66-year-old man presented with dysphagia and dysarthria, and was diagnosed with tongue cancer (cT2N0MX). Initially, the patient's ECOG PS score was 1. He had a past history of external radiation therapy to the chest to treat thoracic esophageal cancer (squamous cell carcinoma, cT3N1M1; M1, brain and bone) and was receiving chemotherapy. He was not considered a candidate for conventional radical therapy for tongue cancer, and therefore he received palliative brachytherapy to relieve the dysphagia.

The tumor was located in the midline on the dorsum of tongue. After local anesthesia, 8 applicators were placed inside the tumor following which, single-fraction brachytherapy was applied.

The size of the lesion decreased, and he was able to eat orally 2 weeks after brachytherapy. The lesion continued to shrink until 10 weeks after treatment (Figure 1). The symptoms of dysphagia and dysarthria continued to diminish until his death. There were no major complications such as oral mucositis. At 24 weeks after the treatment, he had a cerebral infarction, and his performance status deteriorated. The patient died 28 weeks after the treatment.

\section{Patient 2}

A 17-year-old male adolescent presented with oral discomfort and difficulty in mastication, and was diagnosed with right gingival metastasis of osteosarcoma. His initial ECOG PS score was 2. Previously, he had osteosarcoma in left humerus and had undergone chemotherapy and surgery. Later, he developed multiple bone metastases and multiple lung metastases, and clinically no effective chemotherapy was left at the time. At one point, the gingival metastasis had been resected, but grew to previous size within a month. Re-resection was not considered, so he received palliative brachytherapy for the right gingival metastasis in order to provide symptomatic relief.

The tumor was located in the right gingiva behind the molar. After local anesthesia, 4 applicators were placed inside the tumor and then, single-fraction brachytherapy was performed.
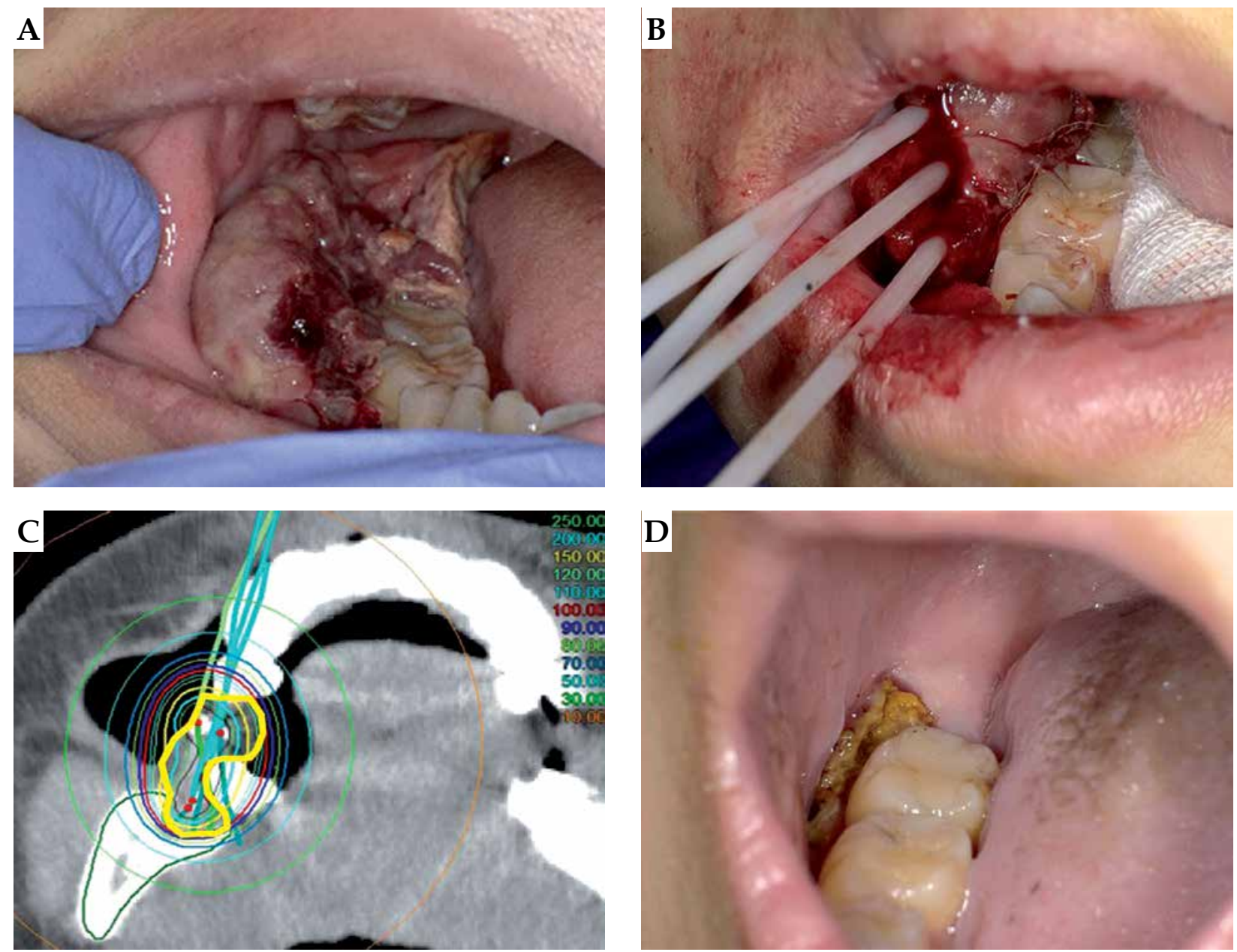

Fig. 2. Patient 2. A) Tumor on the right gingiva; B) Four applicators placed; C) Yellow circle indicates the CTV; D) Tumor at 5 weeks after the treatment 
His symptoms started to diminish 1 week after the treatment. At the same time, he developed grade 1 mucositis (according to CTCAE ver. 4), but this toxicity did not affect his performance status. After the treatment, partial resection of tumor was performed, as he hoped that the additional resection before the lesion shrinkage would prevent him from bite into it at every meal. The symptoms continued to improve and were controlled until his death 28 weeks after the treatment (Figure 2).

\section{Patient 3}

An 88-year-old man presented with deafness, and was diagnosed with carcinoma in the left external acoustic meatus. Initially, ECOG PS score was 2 . He was deaf in the contralateral ear due to chronic otitis media and had right hemiplegia due to a past history of cerebral infarction. Surgery was indicated but declined by the patient. Also, stereotactic radiotherapy was declined as he was not able to maintain the same posture during the treatment. External radiation therapy and fractionated HDR brachytherapy in full doses were refused, because he did not want to receive therapies, which needed outpatient visits for several times. That is why he received palliative brachytherapy in order to improve hearing in his left ear.
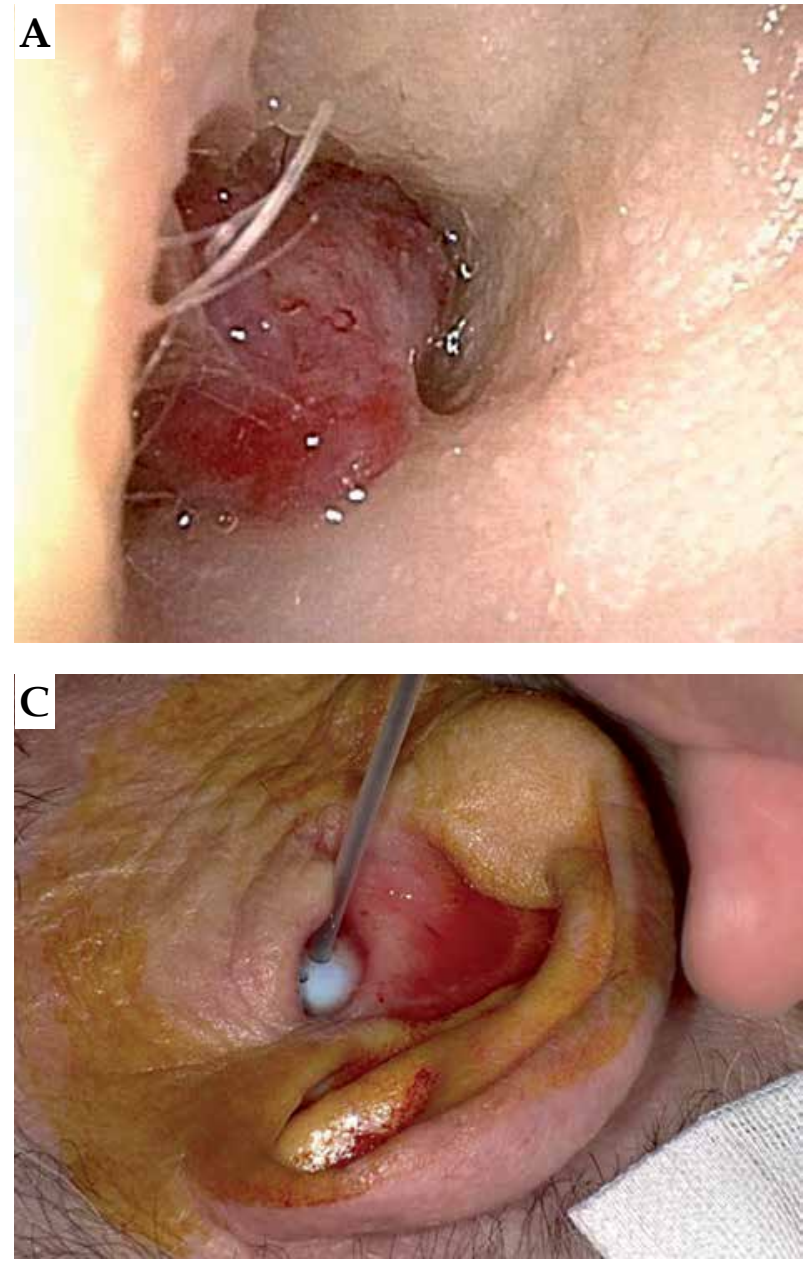

Fig. 3. Patient 3. A) Direct view of the tumor; B) Pretreatment CT image showing the tumor occupying the external auditory canal; C) Applicator in the right external auditory canal; D) Yellow circle indicates the CTV
The tumor had filled the entire left external auditory canal. For local anesthesia, topical 1\% lidocaine gel was applied into the left auditory canal. One applicator was placed inside the tumor, after which single-fraction brachytherapy was performed (Figure 3).

At 5 weeks after treatment, hearing returned in the patient's left ear. Audiometry showed an improvement of auditory activity in left ear (Figure 4). This improvement continued until 39 weeks after treatment. During observation period, no major complications occurred. The tumor later relapsed, but the patient declined re-treatment and died 65 weeks after the treatment.

\section{Discussion}

Palliative HDR brachytherapy successfully improved symptoms in all 3 patients, with improvement starting to be observed within 1-5 weeks after the treatment. Only 1 patient experienced an adverse event (grade 1 oral mucositis).

The prognosis of patients with head and neck cancer with no indication for definitive treatment is generally poor. In such patients, chemotherapy is considered a good treatment option for effective tumor control, and long-term progression-free survival may be possible [9].
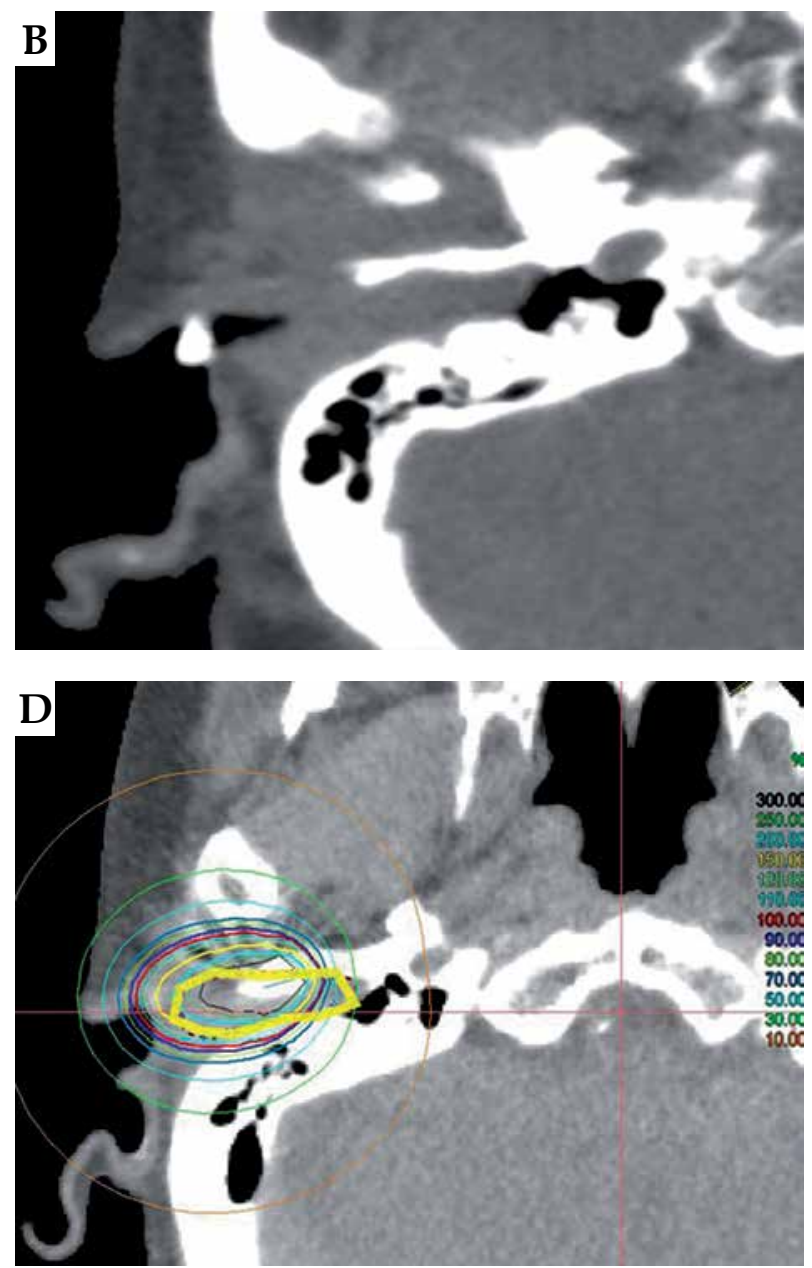
Moreover, quality of life issues generally predominates. Palliative treatment is often needed in such patients as they experience serious difficulties in swallowing, chewing, talking, and cosmetic defects.

For an inoperable disease, palliative external beam radiotherapy (EBRT) has been attempted, but it often requires several sessions, and the treatment is time-consuming. Considering the limited prognosis of these patients, long period of treatment and time to achieve therapeutic effects are the major problems. Compared with EBRT, one main advantage of brachytherapy is that it delivers maximum radiation dose directly to the tumor without damaging healthy surrounding tissues. In particular, HDR brachytherapy can deliver higher dose to the tumor in only couple of minutes.

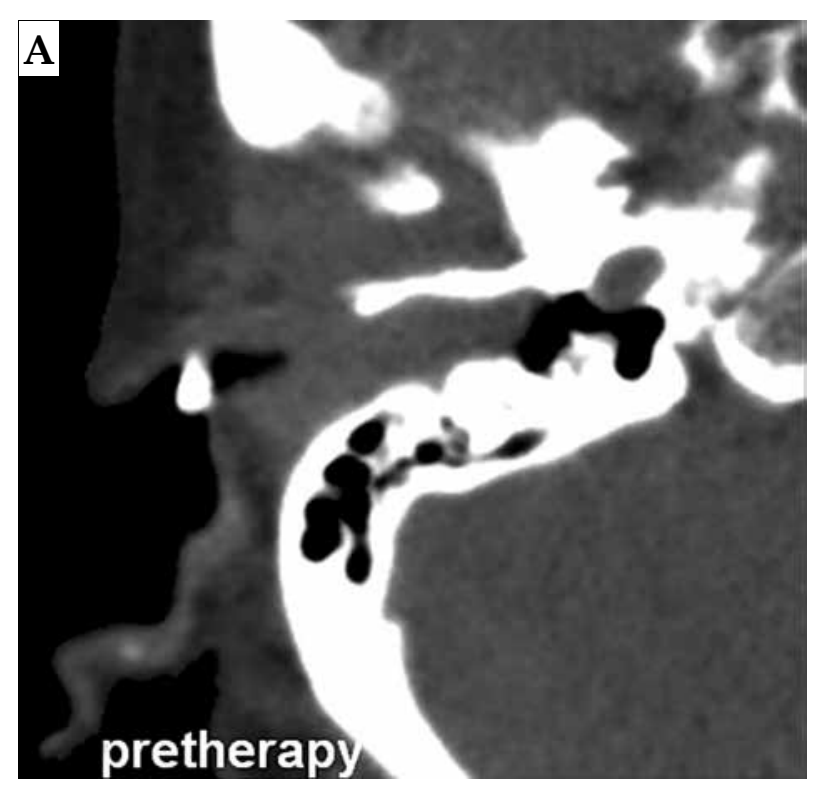

C

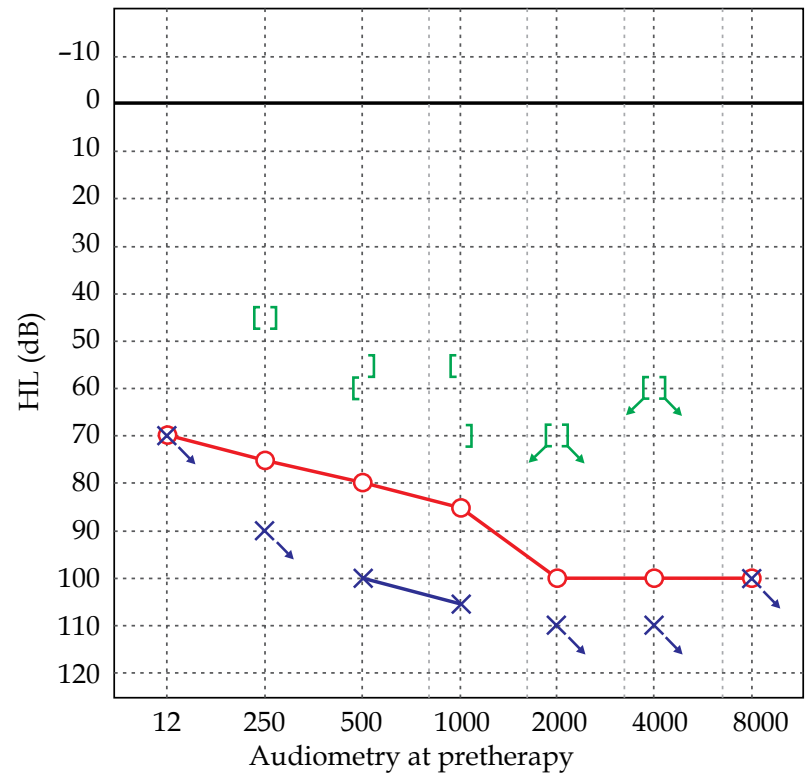

The dose ranging from 15 to 20 Gy is generally used as a single-fraction HDR brachytherapy to liver, kidney, lung, and breast malignancies $[10,11,12,13]$. However, an appropriate single-fraction dose for head and neck cancer in palliative therapy is unknown. We used a single HDR dose of $10 \mathrm{~Gy}$ for our patients by referring to tolerance of OARs and the dose of palliative EBRT, in which $20 \mathrm{~Gy}$ in 5 fractions is commonly used. In terms of $\mathrm{EQD}_{2}$ calculation $(\alpha / \beta=3), 10$ Gy single dose and $20 \mathrm{~Gy}$ in 5 fractions are almost comparable (26 Gy and $28 \mathrm{~Gy}$, respectively).

There are few previous reports on the use of HDR brachytherapy as palliative treatment for head and neck cancer $[6,7,8]$. Skowronek et al. reported the results of palliative HDR and pulsed-dose-rate (PDR) brachytherapy in 47 cases, with a relapse of head and neck cancer [8].

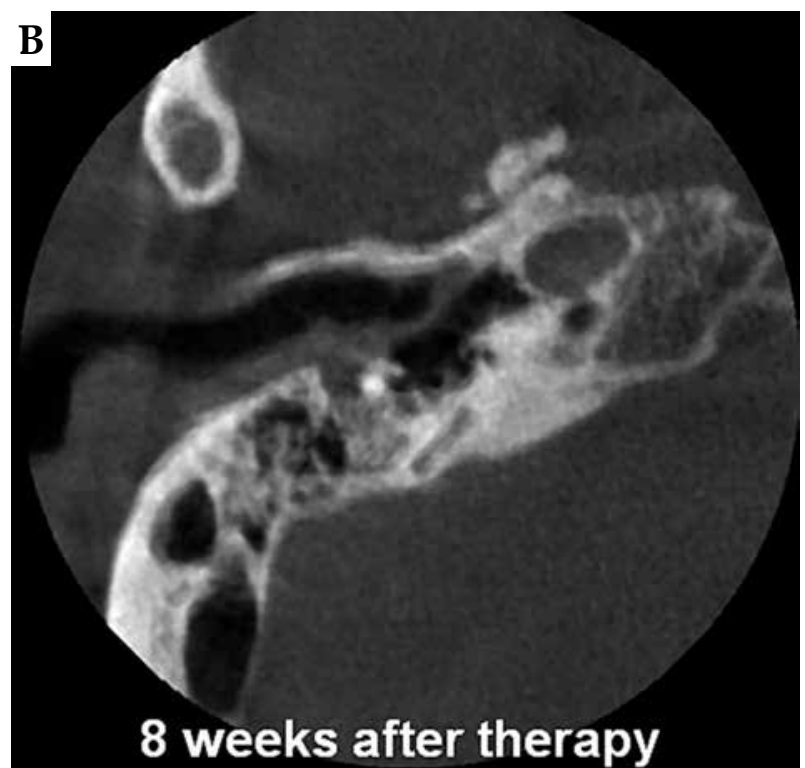

D

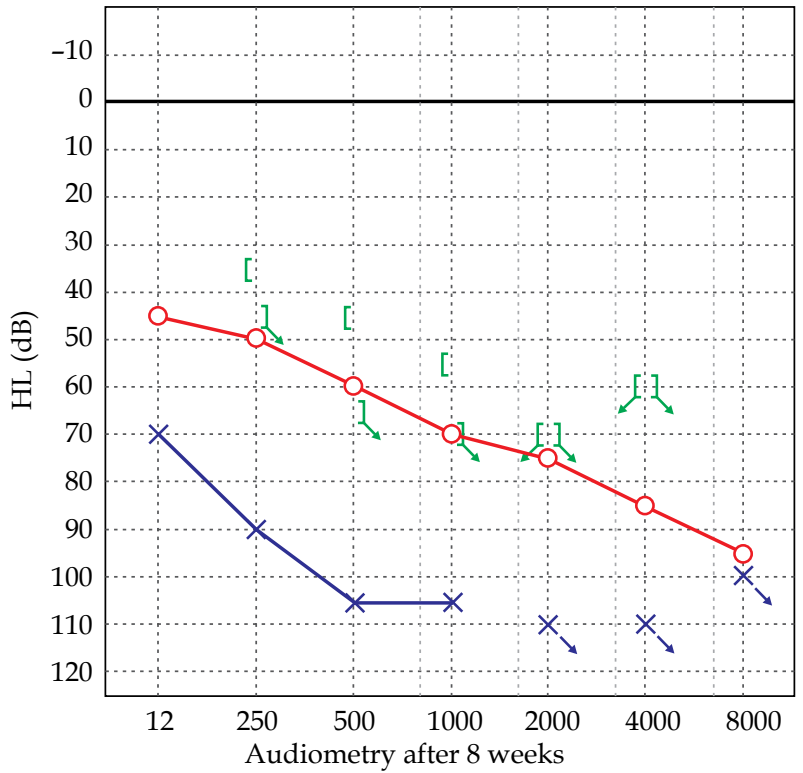

Fig. 4. Patient 3. A, B) CT images show shrinkage of the tumor and patency of the external auditory canal; C, D) Audiometry shows improved hearing at 8 weeks after the treatment 
Thirty-four patients were treated using HDR brachytherapy with 5 doses of $4 \mathrm{~Gy}$ per fraction, and 13 patients were treated using PDR brachytherapy with a total dose ranging from 20 to $40 \mathrm{~Gy}$. The overall response rates at 3 and 6 months after treatment were $64 \%$ and $43 \%$, respectively. In 35 cases $(74.5 \%)$, superficial necrosis was observed within 6 months. In a more recent retrospective study by Bartochowska et al. [6], 156 patients with local or regional recurrence of head and neck cancer were treated with HDR brachytherapy or PDR brachytherapy as palliative treatment. The median total dose of PDR brachytherapy was 20 Gy. HDR brachytherapy was delivered twice a day, with intervals of at least $6 \mathrm{~h}$. The total dose ranged from 12 Gy to $30 \mathrm{~Gy}$, given in 3 to 10 fractions, with 3-6 Gy per fraction over 2-5 days. In that study, the overall response rate at 6 months after treatment was 37.7\%. The overall rate of complications was $35 \%$. In these past reports, response rates were unsatisfactory, and complication rates were relatively high as compared to our cases. We experienced only one case of grade 1 mucositis with palliative effects for all cases. Considering our results, it is possible that the dose of $10 \mathrm{~Gy}$ and CT-based treatment planning contribute to a decrease of complication rate, and 10 Gy is sufficient for palliative effects; although, we could not evaluate an adequate late complications for patients with limited prognosis.

Our treatment technique performed 7-9 months of palliative effects with single-fraction of $10 \mathrm{~Gy}$. Based on our results, our technique can be effective for patients with head and neck cancer who are considered to have approximately half a year of prognosis. However, we experienced a recurrence in patient 3 . Possibly, a dose should be increased when a patient's prognosis is estimated to be more than half a year.

\section{Conclusions}

To our knowledge, this is the first report of single-fraction image-guided HDR palliative brachytherapy for head and neck cancer. Our findings suggest that single-fraction 10 Gy image-guided HDR brachytherapy can be a safe and effective palliative treatment, and may be a treatment option for symptomatic patients with inoperable head and neck cancer.

\section{Disclosure}

The authors report no conflict of interest.

\section{References}

1. Nag S, Kelly JF, Horton JL et al. The American Brachytherapy Society recommendations for HDR brachytherapy for carcinoma of the lung. Oncology 2001; 15: 371-381.

2. Mazeron JJ, Ardiet JM, Haie-Méder et al. GEC-ESTRO recommendations for brachytherapy for head and neck squamous cell carcinomas. Radiother Oncol 2009; 91: 150-156.

3. Kovács G, Martinez-Monge R, Budrukkar A et al. GEC-ESTRO ACROP recommendations for head \& neck brachytherapy in squamous cell carcinomas: 1st update - Improvement by cross sectional imaging based treatment planning and stepping source technology. Radiother Oncol 2016; 122: 248-254.
4. Lukens JN, Gamez M, Hu K et al. Modern brachytherapy. Semin Oncol 2014; 41: 831-847.

5. Suzuki G, Hayabuchi N, Kurata S et al. Early-stage carcinoma of the external auditory canal treated by intracavitary irradiation with HDR 192Ir-RALS: a case report. Nihon Igaku Houshasen Gakkai Zasshi 2004; 64: 398-400.

6. Bartochowska A, Wierzbicka M, Skowronek J et al. Highdose-rate and pulsed-dose-rate brachytherapy in palliative treatment of head and neck cancers. Brachytherapy 2012; 11: 137-143.

7. Tselis N, Karagiannis E, Kolotas C et al. Image-guided interstitial high-dose-rate brachytherapy in the treatment of inoperable recurrent head and neck malignancies: An effective option of reirradiation. Head Neck 2017; 39: E61-E68.

8. Skowronek J, Wierzbicka M, Milecki P et al. Palliative HDR and PDR brachytherapy in treatment of head and neck cancer relapses. Otolaryngol Pol 2004; 58: 721-729.

9. Fury MG, Pfister DG. Current recommendations for systemic therapy of recurrent and/or metastatic head and neck squamous cell cancer. J Natl Compr Canc Netw 2011; 9: 681-690.

10. Kovács A, Iezzi R, Cellini F et al. Critical review of multidisciplinary non-surgical local interventional ablation techniques in primary or secondary liver malignancies. J Contemp Brachytherapy 2019; 11: 589-600.

11. Ricke J, Wust P, Wieners G et al. CT-guided interstitial single fraction brachytherapy of lung tumors: phase I results of a novel technique. Chest 2005; 127: 2237-2242.

12. Kishan AU, Lee EW, McWilliams J et al. Image-guided highdose-rate brachytherapy: preliminary outcomes and toxicity of a joint interventional radiology and radiation oncology technique for achieving local control in challenging cases. J Contemp Brachytherapy 2015; 7: 327-335.

13. Kinj R, Chand ME, Gal J et al. Five-year oncological outcome after a single fraction of accelerated partial breast irradiation in the elderly. Radiat Oncol 2019; 14: 234. 